Musées, Patrimoine et Culture scientifiques et techniques

$148 \mid 2013$

juillet-août 2013

\title{
Étude et restauration de la momie égyptienne du muséum de Nantes
}

\section{Marie-Laure Guérin}

\section{(Q) OpenEdition \\ Journals}

Édition électronique

URL : http://journals.openedition.org/ocim/1241

DOI : 10.4000/ocim. 1241

ISSN : 2108-646X

Éditeur

OCIM

Édition imprimée

Date de publication : 10 juillet 2013

Pagination : $12-20$

ISSN : 0994-1908

Référence électronique

Marie-Laure Guérin, "Étude et restauration de la momie égyptienne du muséum de Nantes », La Lettre de I'OCIM [En ligne], 148 | 2013, mis en ligne le 01 juillet 2015, consulté le 19 avril 2019. URL : http:// journals.openedition.org/ocim/1241; DOI : 10.4000/ocim.1241 


\section{Étude et restauration}

\section{de la momie égyptienne du muséum de Nantes}

\section{Marie-Laure Guérin*, Philippe Mainterot, Alexandre Brunet, Juliette Langlois, Nathalie Gandolfo, Pascale Richardin, Laure Cadot et Noëlle Timbart}

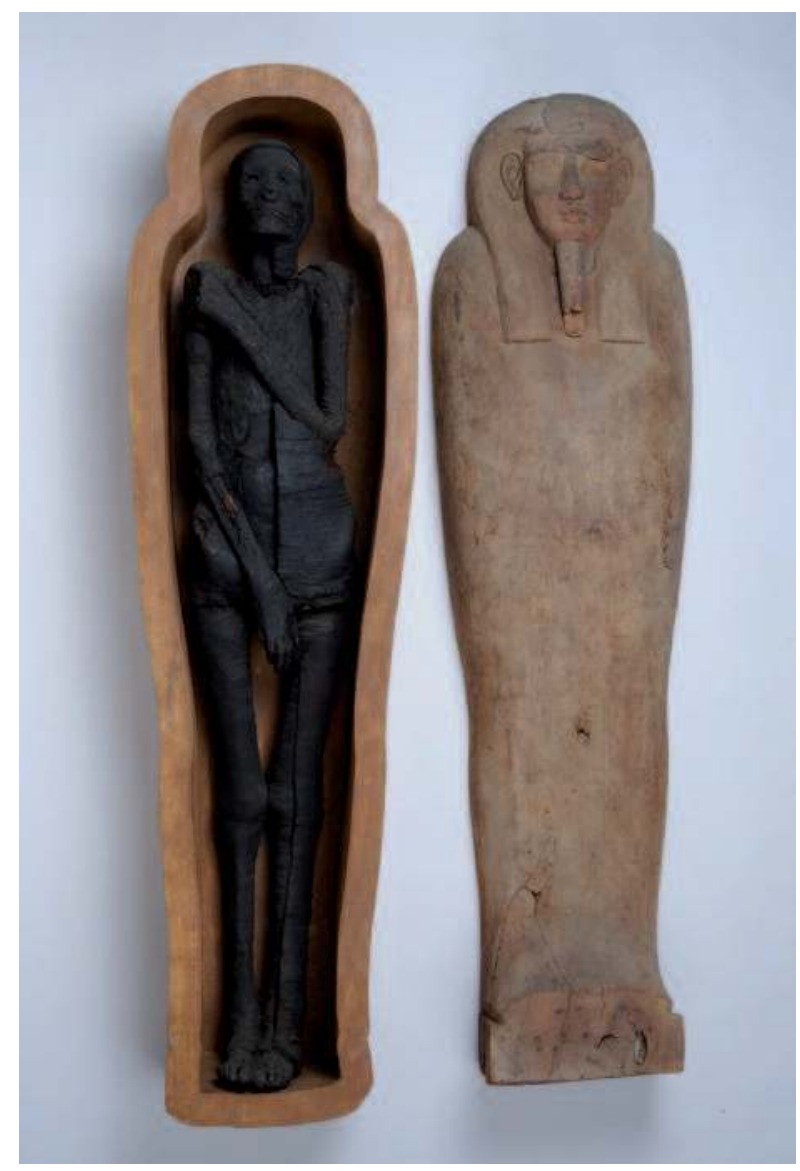

La momie et le couvercle de sarcophage (c) Patrick Jean

* Marie-Laure Guérin, qui a coordonné cet article, est chargée de collections au muséum de Nantes marie-laure.guerin@mairie-nantes.fr
Intégrée dans les collections du muséum d'Histoire naturelle de Nantes depuis le début du XIXe siècle, une momie égyptienne a récemment été étudiée, analysée et restaurée par un égyptologue, un médecin radiologiste, des physiciens, des chimistes, des conservateurs et des restaurateurs qui relatent ici les différentes étapes de cette collaboration pluridisciplinaire.

En marge de ses collections d'Histoire naturelle, le muséum de Nantes conserve une collection d'archéologie dans laquelle figurent une momie égyptienne et un couvercle de sarcophage. Ces deux pièces ont été rapportées d'Égypte et données au muséum par Frédéric Cailliaud en 1819.

Malheureusement, le donateur n'a laissé aucune documentation à leur sujet : la provenance géographique précise et les circonstances de la collecte de ces pièces sont par conséquent inconnues ; on ignore également si le couvercle et la momie étaient associés à l'origine. Alors que le corps de la momie est partiellement débarrassé de ses bandelettes, aucune archive ne fait référence à cet épisode pas plus qu'à une éventuelle étude. Enfin, le sarcophage dont le décor aurait pu fournir des indications a été décapé à une époque indéterminée. En 2004, suite à la rénovation de la salle où il était exposé, cet ensemble, en marge des collections du musée, est placé dans les réserves.

La momie devient l'objet d'un regain d'intérêt grâce à l'étude qu'en fait Philippe Mainterot qui rédige en 2008 sa thèse sur les voyages et les collections de Frédéric Cailliaud. Les hypothèses qu'il soulève nous incitent à nous rapprocher d'autres personnes compétentes dans l'étude de ces restes humains. 
Pour cela, il est nécessaire d'engager préalablement une opération de conservation-restauration car la momie doit être manipulée et déplacée. La construction récente de réserves muséales modernes nous donne l'assurance qu'elle sera placée dans de bonnes conditions de conservation après traitement et conditionnement.

Depuis lors, un égyptologue, un médecin radiologiste, des conservateurs, des restaurateurs et des physicochimistes se sont succédés auprès de la momie du muséum de Nantes pour l'étudier, l'analyser et mieux la conserver. C'est cette collaboration que nous nous proposons de rapporter dans cet article.

\section{Les voyages ef les collections de Frédéric Cailliaud}

(Philippe Mainterot, égyptologue, université de Nantes, CRHI-EA 1163)

Le voyageur nantais Frédéric Cailliaud (1787-1869) est à placer au rang des pionniers de l'égyptologie. Cette discipline s'est forgée dans les deux premières décennies du XIXe siècle à la suite de l'Expédition d'Égypte, avec comme point d'orgue le déchiffrement du système hiéroglyphique par Jean-François Champollion en $1822^{(1)}$.

Entre 1815 et 1818, Cailliaud parcourut l'Égypte au fil du Nil et se lança dans l'exploration des déserts orientaux et occidentaux, mettant ainsi au jour bon nombre de temples égyptiens jusqu'alors inconnus. De retour en France à la fin de l'année 1818, Frédéric Cailliaud fut presque aussitôt renvoyé en Égypte par le gouvernement de Louis XVIII et l'Académie des Inscriptions et Belles-Lettres, cette fois dans le cadre d'une mission scientifique officielle : poursuivre l'exploration de l'Égypte au-delà de l'île de Philae, où les savants de Bonaparte s'étaient arrêtés en 1801. Il était accompagné d'un jeune aspirant de marine, Pierre-Constant Letorzec, pour le seconder dans la réalisation d'une nouvelle cartographie du pays. Ce fut au cours de ce nouveau périple qu'il parvint jusqu'à l'antique cité de Méroé, la capitale de l'ancien royaume de Koush, et mit au jour sa nécropole à pyramides en avril 1821 .

Parallèlement à ses explorations, le voyageur constitua une collection d'antiquités pharaoniques, à l'image de beaucoup d'Européens qui commençaient à visiter les rives du Nil au début du XIX ${ }^{\mathrm{e}}$ siècle. Ces objets, ainsi que les notes et relevés du voyageur furent en grande partie acquis par le gouvernement lors de son retour en France, en octobre 1822. Le récit de son premier séjour en Égypte (1815-1818) fut publié par l'académicien Edme-François Jomard sous le titre Voyage à l'Oasis de Thèbes en 1822, puis en 1862 ; tandis que
Cailliaud édita seul son récit du second voyage avec l'aide de l'Imprimerie Royale entre 1826 et 1827 : le Voyage à Méroé et au Fleuve Blanc.

À partir de l'étude des documents que nous avons rassemblés après enquête ${ }^{(2)}$, cette momie et son cercueil furent rapportés par Frédéric Cailliaud au terme de son premier séjour égyptien en 1818. Il s'agit des seules informations dont nous disposons les concernant. Après avoir vendu la majeure partie de sa collection au gouvernement, Cailliaud revint parmi les siens à Nantes en mai 1819. Nous avons démontré qu'il avait alors conservé quelques antiquités pharaoniques qui devaient former sa collection particulière. Cailliaud décida alors d'offrir au muséum d'Histoire naturelle de Nantes une quarantaine d'objets égyptiens que le conservateur de l'époque, François-René Dubuisson, présenta dans un article du Journal de Nantes et de la Loire-Inférieure, en date du 18 juin

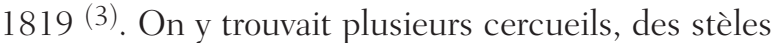
et des serviteurs funéraires. À l'exception de cette momie et du couvercle du cercueil, toutes ces pièces furent mises en dépôt en 1856 au musée de la Société Archéologique de Nantes et font aujourd'hui partie des collections du musée départemental Thomas Dobrée.

La particularité de cette momie réside dans le fait qu'elle a été partiellement démaillotée au XIXe siècle pour être étudiée. En effet, toute la partie droite du corps a été retirée des différentes couches de bandelettes collées entre-elles au moyen d'un baume, y compris sur la face, afin d'atteindre les tissus organiques et le squelette. D’autres momies rapportées d'Égypte par Frédéric Cailliaud subirent également ce traitement à l'occasion de « séances publiques » très bien documentées. À titre d'exemples, on peut citer l'ouverture de la momie de Padiimenipet, fils de Sôter, par Cailliaud lui-même devant la haute société parisienne le 30 novembre 1823 ; ou bien de celle qui fut offerte à la Société Académique de Nantes, débandelettée en 1826, qui fut à l'époque l'objet d'un rapport complet du Docteur Maréschal dans le Journal de Médecine ${ }^{(4)}$. Nous avons également la trace d'une autre ouverture de momie réalisée par Cailliaud le 10 septembre 1843 à l'occasion du Congrès scientifique de France qui se tint exceptionnellement à la Société des Beaux-Arts de Nantes.

Le couvercle du cercueil accompagnant cette momie est quant à lui beaucoup plus énigmatique. Un examen attentif de sa surface montre qu'il a été soigneusement décapé. Toutefois, certaines traces du décor subsistent sur la partie extérieure, alors que la partie intérieure 
était dénuée de toute représentation et taillée grossièrement. D'aspect momiforme, il est constitué de plusieurs éléments assemblés au moyen de tenons. La tête est coiffée d'une perruque striée longitudinalement, laissant les oreilles dégagées. Le visage se caractérise par de grands yeux stylisés en relief, un nez aux narines ouvertes et une bouche charnue. Une barbe postiche vient s'encastrer dans le menton, mais son extrémité est brisée au niveau de la courbure. Quelques traces d'un grand collier ousekh polychrome sont encore visibles sur la poitrine, au-dessus de la déesse du ciel Nout, représentée agenouillée, étendant ses ailes sur le corps du défunt. Sur la partie inférieure, on distingue également plusieurs colonnes de texte hiéroglyphique. Ces inscriptions demeurent malheureusement indéchiffrables ${ }^{(5)}$; elles auraient pu nous renseigner sur l'identité de son propriétaire. Toutefois, cette organisation typique du décor, l'organisation des formules rituelles en colonnes sur les jambes et la forme globale du cercueil renvoient stylistiquement à la BasseÉpoque, soit aux alentours de 750 à 332 av. J.-C.

La présence de ces deux éléments dans les collections Cailliaud laisse en suspend la question de leur origine géographique précise. En reprenant les journaux de voyage de Cailliaud, ainsi que les inventaires détaillés de ses collections, on remarque que les équipements funéraires comprenant des momies furent découverts soit dans la nécropole thébaine, soit dans celle de Memphis, à proximité du site de Saqqâra. Ce fut très probablement le cas de celle-ci. Son exposition dans les salles du muséum d'Histoire naturelle de Nantes serait attestée depuis le XIXe siècle, notamment à travers le témoignage de Gustave Flaubert qui put observer en 1847 une "momie égyptienne, debout, à côté de son cercueil peint» (6). Selon une autre hypothèse, il pourrait s'agir du cercueil de la chanteuse d'Amon-Rê Ankhoririou, présent dans les collections du muséum jusqu'en 1857, qui se trouve aujourd'hui conservé au musée Dobrée et qui a, quant à lui, conservé sa polychromie. Considérant le manque d'éléments historiographiques précis de cet ensemble, il sera difficile de privilégier l'une de ces deux pistes par rapport à l'autre.

\section{Contexte muséographique}

(Marie-Laure Guérin, chargée de collections

au muséum de Nantes)

Lorsque Gustave Flaubert visita le muséum de Nantes, celui-ci occupait l'ancienne école de chirurgie située au bord de la rivière Erdre et était décrit par ceux qui le fréquentaient comme un endroit humide et exigu. Frédéric Cailliaud qui en était devenu le directeur, obtint de la municipalité la construction d'un nouveau musée à l'emplacement actuel. Cailliaud décéda au cours des travaux et c'est son successeur, Édouard Dufour qui se chargea de la mise en place des collections. L'inauguration eut lieu le 19 août 1875.

Parmi les différentes descriptions du musée qui ont suivi l'inauguration, ce n'est qu'en 1924, que l'on trouve une évocation de la momie. Dans le guide du visiteur, Ernest Marchand, directeur de 1920 à 1933, situe la momie au premier étage, dans la première vitrine en commençant par la gauche.

Elle est restée dans cette vitrine jusqu'en 2004. Elle était présentée debout à côté d'autres restes humains : une tête de guerrier Maori, aujourd'hui restituée à la Nouvelle Zélande, une tête momifiée provenant d'un caveau et une peau d'homme tannée.

Dans les années 1970, suite à la rénovation de la salle, la momie est placée dans un faux sarcophage construit pour l'occasion et toujours présentée verticalement. Nous verrons par la suite de l'étude les conséquences néfastes de cette présentation verticale.

À partir de 2010, il fut décidé d'étudier et de restaurer la momie et son sarcophage. Sur les conseils du Laboratoire Arc'Antique, une collaboration a pu être établie avec le CHU de Nantes (protocole d'accord) afin de procéder à une scannographie.

Une radiographie préalable de la momie dans son cercueil a révélé que la momie était fixée au fond à l'aide de 6 vis modernes et que la tête était maintenue au tronc par la présence d'une tige métallique. De plus, de nombreux clous présents dans le cercueil étaient incompatibles avec une bonne imagerie. Il fallait donc désolidariser et sortir la momie de son coffrage pour procéder à la scannographie. Cette opération délicate a été réalisée avec l'aide de 5 personnes, un plateau de manipulation a été glissé sous la momie et celle-ci remise dans le cercueil qui demeurait nécessaire pour le transport.

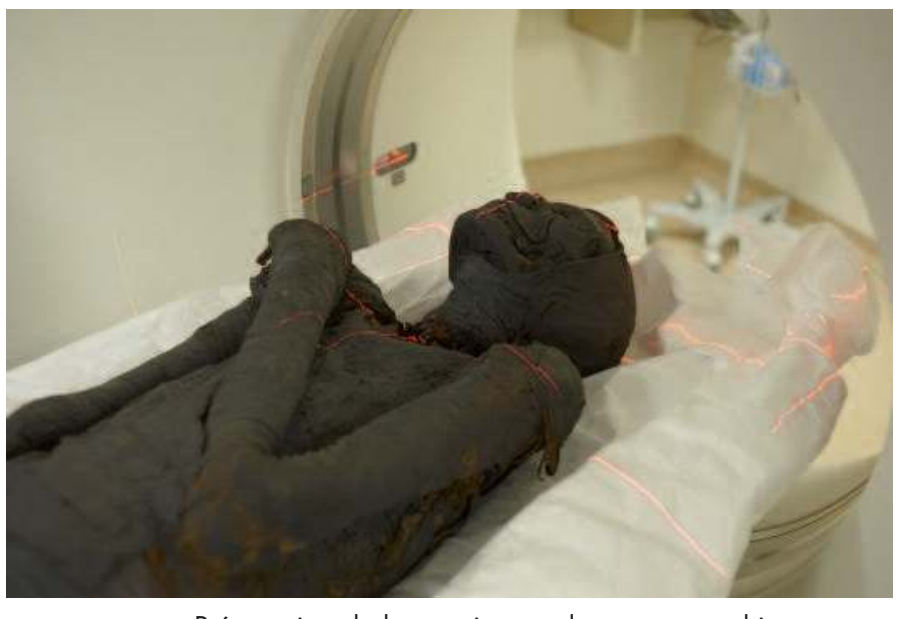

Préparation de la momie pour la scannographie (c) Jean-Gabriel Aubert 


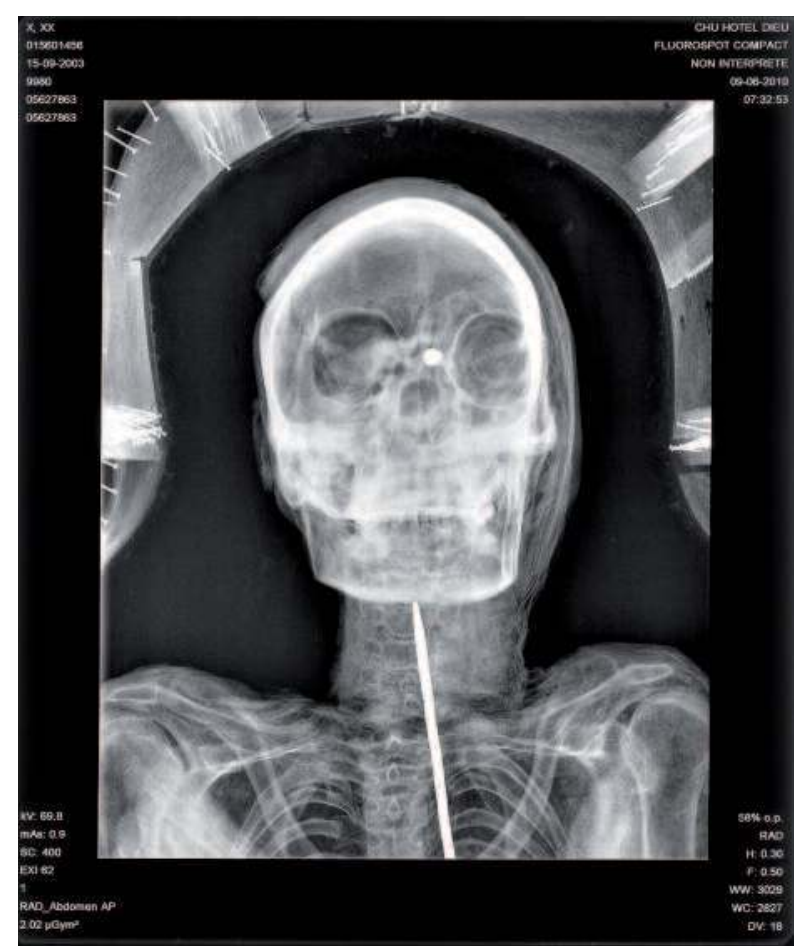

Radiographie de la tête de la momie (-) $\mathrm{CHU}$ de Nantes

Le Centre de Recherche et de Restauration des Musées de France (C2RMF) et des conservateurs-restaurateurs ont été contactés pour entreprendre le projet de conservation-restauration. Une anoxie dynamique à flux d'azote continu a été réalisée préalablement à leurs interventions afin d'éliminer une infestation biologique avérée.

Le sarcophage quant à lui a été photographié sous lumières ultraviolette et infrarouge par le Laboratoire Arc'Antique dans l'espoir de révéler les inscriptions qui avaient été effacées et que l'on devinait encore. Malheureusement, cette opération a donné peu de résultats.

\section{Ce que révèlent la radiographie et la scanographie de la momie}

(Alexandre Brunet, radiologue au service d'imagerie médicale du CHU de Nantes)

L'imagerie médicale permet, comme sur le vivant, une étude non invasive, c'est-à-dire « sans ouvrir », du contenu interne de la momie. La scannographie est aujourd'hui complémentaires des radiographies standard, elle présente un double avantage : l'obtention de coupes très fines par les scanners modernes permet la reconstruction et la visualisation d'images dans tous les plans, ainsi que des reconstructions tridimensionnelles; et l'analyse des structures «molles », non osseuses.

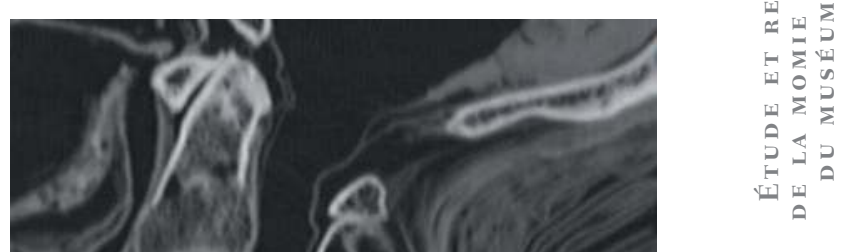

Arthrose cervicale débutante avec pincement discal (flèche pleine). À noter la très bonne visibilité de la dure-mère entourant la moelle épinière (qui n'est elle plus visible, flèche en pointillés).

On retrouve plus bas la rupture de la colonne vertébrale liée au détachement de la tête. (c) $\mathrm{CHU}$ de Nantes

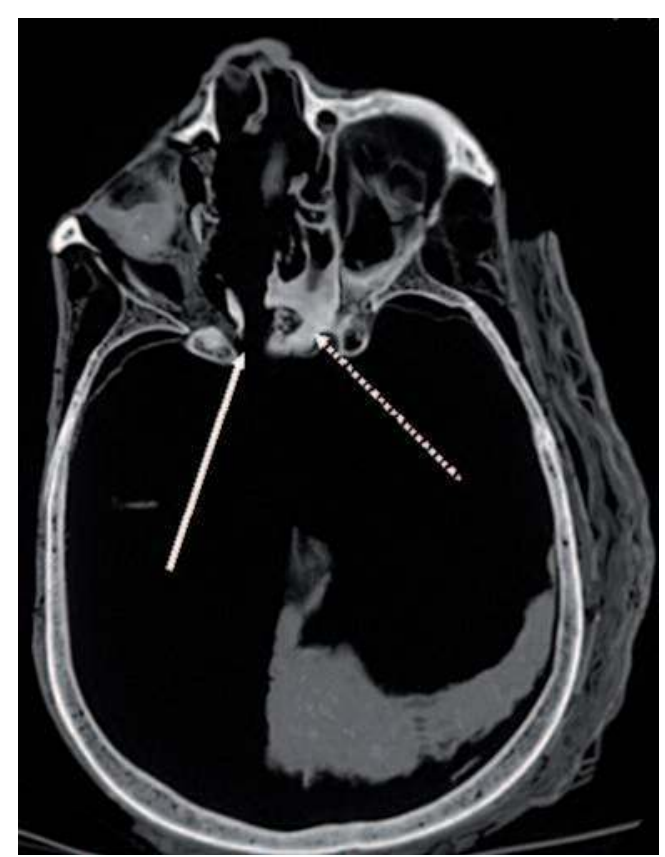

Coupe axiale du crâne montrant l'orifice non naturel créé pour extraire le cerveau (flèche pleine) au sein d'un important épaississement osseux de la paroi des sinus sphénoïdaux (flèche en pointillés),

normalement d'épaisseur infra millimétrique, témoignant d'une sinusite chronique. La masse sombre en arrière de la boîte crânienne correspond vraisemblablement à une résine d'embaumement; on y distingue nettement la trace récente du pas de la vis qui maintenait la tête au fond du cercueil de présentation. (๑) $\mathrm{CHU}$ de Nantes 
Une acquisition corps entier a été réalisée en 2010 sur un appareil Lightspeed 64 barettes de General Electrics (Milwaukee, États-Unis) mis en service en 2007, les images ont été reconstruites à l'aide d'un filtre dur, pour étudier les structures osseuses, et un filtre mou, pour l'analyse des structures non osseuses. Ce qui ressort en premier lieu est le bon état de conservation de l'ensemble des structures osseuses, qui permet de tirer plusieurs conclusions :

- la largeur du bassin permet de dire qu'il s'agit d'une femme ;

- l'ensemble des cartilages de croissance étant soudés la croissance était terminée, ce qui permet de lui donner un âge d'au moins 20 ans ;

- on remarque quelques lésions dégénératives d'arthrose rachidienne cervicale, peu nombreuses. Il existe bien sûr des susceptibilités individuelles intrinsèques (génétiques) et extrinsèques (activités physiques, profession) au développement de telles lésions, mais celles-ci sont quasi-inexistantes avant 30 ans.

La constatation de lésions arthrosiques est extrêmement banale, mais l'analyse du squelette permet dans certains cas de retrouver des signes en faveur de certaines affections chroniques infectieuses (syphilis tertiaire, tuberculose), cancéreuses (métastases osseuses), métaboliques (rachitisme, affections endocriniennes...) ce qui n'est pas le cas ici.

Une autre «curiosité » osseuse consiste en un important épaississement des parois osseuses des sinus aériens de la face, notamment les sinus sphénoïdaux, ce qui témoigne d'une sinusite chronique. On voit également sur ces mêmes sinus l'orifice créé pour extraire le cerveau par voie nasale avant l'embaumement (l'épaisseur des parois osseuses a pu peut-être entraîner quelques difficultés à la perforation, celleci étant habituellement très fine, millimétrique)

L'ensemble des viscères ayant été extraits au moment de la momification, ils ne sont bien sûr pas retrouvés sur le scanner, néanmoins, on remarque que les structures très fibreuses, comme les ligaments ou les ménisques, sont très bien conservés car parfaitement visibles. Il en est de même de la dure-mère, la plus externe et la plus fibreuse des enveloppes méningées.

\section{Datation par le carbone-14 et analyses physico-chimiques}

(Pascale Richardin, ingénieure de recherche au C2RMF ;

Nathalie Gandolfo et Juliette Langlois, assistantes ingénieurs au C2RMF)

La datation des momies est essentielle afin de replacer les individus dans un cadre chronologique et culturel fiable, en particulier pour des momies conservées dans des musées, dont les origines archéologiques ou

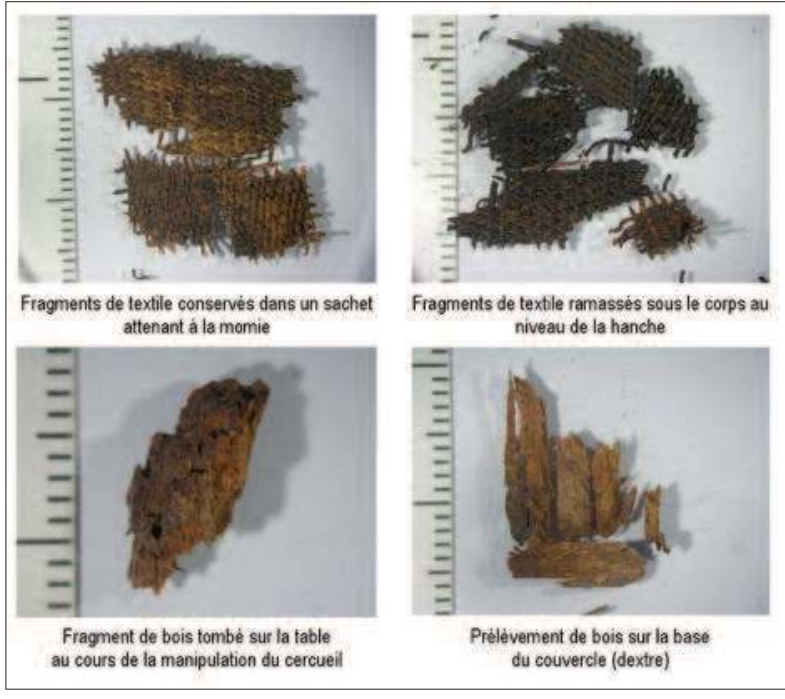

Photographies sous loupe binoculaire des 4 échantillons à dater (c) C2RMF/Nathalie Gandolfo

historiques sont parfois peu documentées. En effet, il a été montré que certaines momies n'étaient pas associées aux cercueils ou aux sarcophages dans lesquels elles étaient exposées ${ }^{(7)}$. La datation directe des corps par le carbone-14 est généralement réalisée sur les os (8) ou pour les momies sur les cheveux (9) ou d'autres tissus biologiques (10). Cependant, pour la momie de Nantes, en raison de l'absence de cheveux et afin d'éviter un échantillonnage trop invasif, nous avons prélevé deux échantillons du linceul. Le cercueil a également été daté grâce à deux fragments de bois provenant du couvercle.

Avant toute datation par le carbone-14 d'un textile, il est nécessaire de connaître son origine (animale ou végétale), afin de sélectionner le protocole adéquat. Les analyses par spectroscopie infrarouge des deux textiles ont montré leur origine végétale (lin). Ils seront donc traités comme des échantillons végétaux. Les étapes nécessaires pour la préparation d'un échantillon végétal sont : un prétraitement chimique (protocole classique Acide, Base, Acide (11)), une combustion pour extraire le gaz carbonique et une graphitisation (transformation de ce gaz en carbone graphite). C'est sur ce carbone que sera mesuré le radiocarbone. La mesure du carbone-14 a été réalisée par Spectrométrie de masse par accélérateur (AMS) au Commissariat à l'Énergie Atomique CEA de Saclay (12) (13), selon un protocole bien défini ${ }^{(14)}$. Les âges 14C (ou âge radiocarbone) s'expriment en années BP (Before Present : avant 1950) et la calibration permet de passer d'une date $\mathrm{BP}$ à un intervalle de temps en années réelles (avant ou après J.-C.).

Les âges radiocarbone obtenus (pour le bois et les textiles) s'échelonnent entre 2445 et 2500 ans BP. 
Ces valeurs se trouvent sur un plateau de la courbe de calibration et les plages des dates calendaires sont par conséquent larges (entre 800 et 400 ans av. J.-C.). Les échantillons sont donc de la Basse-Époque, période de l'histoire de l'Égypte antique s'échelonnant de 750 à 30 av. J.-C.

Nous avons prélevé un échantillon de la « colle » utilisée vraisemblablement pour une restauration antérieure au niveau de la cuisse gauche ainsi qu'un échantillon de matière noire recouvrant le linceul au niveau de la hanche et un autre à l'arrière des pieds au niveau des talons. Les trois prélèvements ont été analysés par spectroscopie infrarouge.

Le spectre obtenu pour la colle de restauration montre qu'il s'agit d'une protéine et donc d'une colle de peau. Les deux autres prélèvements présentent des spectres similaires à ceux des gommes végétales. La composition en monosaccharides de base a été étudiée par chromatographie en phase gazeuse (CPG). Cette méthode permet également de caractériser l'espèce (15) (16). Ainsi, le profil chromatographique obtenu pour la matière noire recouvrant le linceul au niveau de la hanche témoigne de la présence d'une gomme arabique, vraisemblablement issue d'un Acacia nilotica (17) (18). La gomme arabique est la plus ancienne et la plus connue de toutes les gommes. Les Égyptiens la nommaient kami et lutilisaient comme adhésif lors du bandelettage des momies (19).

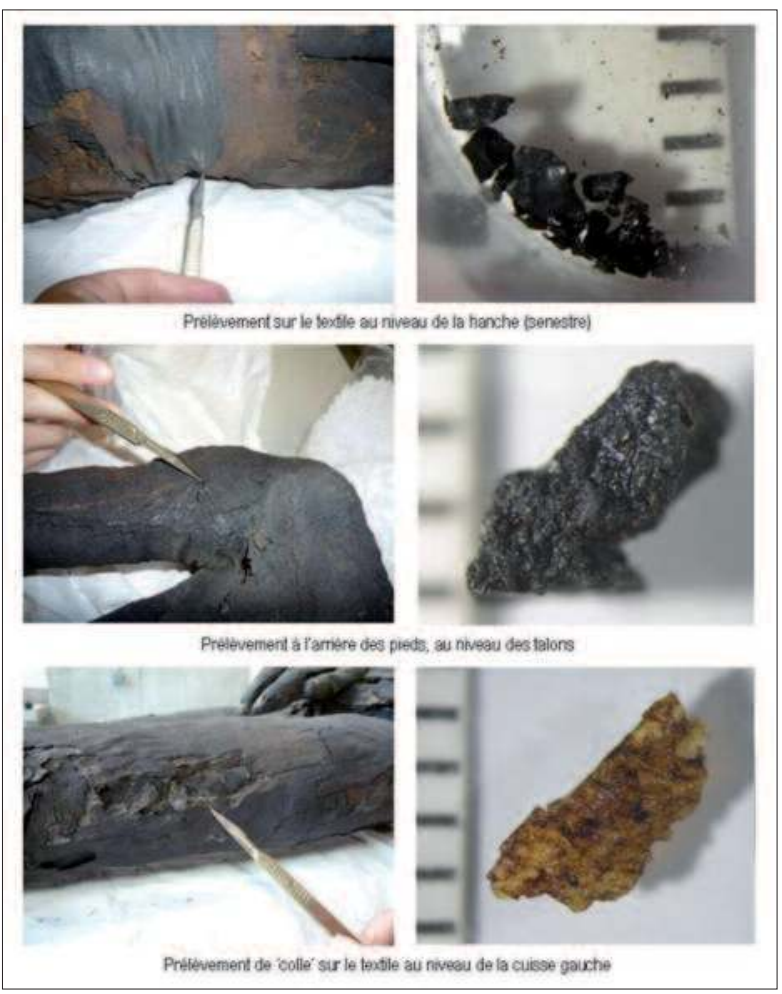

Photographies de la localisation et observation sous loupe binoculaire des 3 échantillons de matière organique (c) C2RMF/Pascale Richardin et Nathalie Gandolfo

\section{Observations macroscopiques et restauration}

(Laure Cadot, restauratrice d'objets ethnographiques et Noëlle Timbart, conservateur au C2RMF)

Dans le cadre de ses missions, le C2RMF a été sollicité par le muséum pour apporter ses conseils et son expertise en matière de conservation et de restauration des restes humains. Une mission menée au muséum le 12 novembre 2010 a permis de réaliser un premier constat d'état de la momie et de déterminer les interventions nécessaires pour assurer sa bonne conservation. À l'issue de cette mission, il a été convenu que la momie serait restaurée sur place, puisque le muséum disposait de suffisamment d'espace pour abriter une telle opération. Noëlle Timbart, conservateur auprès de la filière Archéologie et Ethnographie du département Restauration, chargée des Antiquités égyptiennes et orientales, a assuré le suivi scientifique et technique de la restauration en collaboration avec Laure Cadot, restauratrice d'objets ethnographiques spécialisée dans le domaine des restes humains. Cette opération de conservation s'est déroulée sur deux jours et s'est amorcée par une étape d'observation approfondie afin de mieux comprendre la technique de préparation et de dresser un constat d'état permettant d'établir un diagnostic le plus précis possible quant à l'état de conservation actuel du corps.

\section{Observations macroscopiques}

Le corps est étendu sur le dos, jambes en extension. La tête est légèrement renversée vers l'arrière, le bras dextre est en position tendue, main sur le pubis tandis que la main senestre est ramenée sur l'épaule dextre. Cette position ne semble pas indiquer de rang social particulier ni s'apparenter à un genre plus qu'à un autre et se retrouve assez fréquemment aux époques tardives allant de la troisième période intermédiaire (environ $X^{e}$ siècle av. J.-C.) à la basse époque, voire jusqu'à l'époque ptolémaïque (IVe siècle av. J.-C.) (20). Le découpage méthodique des bandelettes dans le sens longitudinal laisse la moitié supérieure dextre du corps ainsi que le visage, accessible, nous permettant de mieux comprendre les étapes de préparation de la défunte.

L'intégralité du corps a été bandelettée sur des épaisseurs variables - de neuf à dix-huit couches selon les parties du corps - avec des bandes de lin assez fines pouvant s'élargir jusqu'à former des pièces plus importantes au niveau des fesses notamment. On observe la présence de franges à l'extrémité de certaines bandelettes (cheville senestre, cou). Chaque membre a été bandeletté indépendamment avant que le corps 


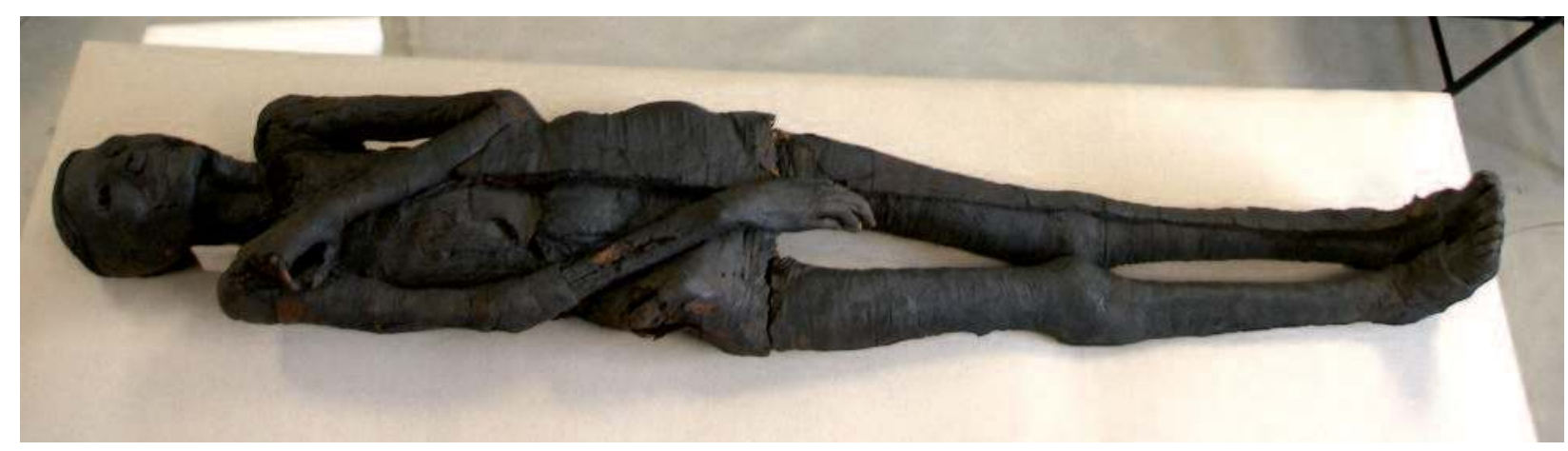

La momie allongée sur le dos () Laure Cadot

ne soit vraisemblablement placé dans un linceul. Chaque couche de textile ainsi que le corps lui-même sont imprégnés d'une résine noire identifiée comme de la gomme végétale, de type gomme arabique (Acacia nilotica). Un tampon de toiles a été introduit dans les narines et vraisemblablement dans les cavités orbitales. Quelques cheveux en début de repousse sont visibles derrière l'oreille dextre. Cet indice semblerait indiquer que le crâne ait été rasé peu de temps avant la mort de la femme ou peut-être au moment de l'embaumement. Aucun autre exemple connu n'autorise de mise en parallèle ou d'interprétation.

Encore très masqué par les restes de bandelettage, il n'a pas été possible d'observer si des traces d'incision étaient visibles sur le buste. Toutefois cette possibilité n'est pas à exclure.

La technique de dessiccation du corps est également difficile à identifier de façon formelle, toutefois, on peut supposer en s'appuyant sur les textes connus (dont Hérodote, Histoires, II, 86) que le corps a été mis en contact avec du natron, sel de carbonate de sodium hydraté, aux propriétés desséchantes.

\section{Altérations-diagnostic}

La momie présente des fragilités évidentes du squelette visibles macroscopiquement ainsi que sur les radiographies entraînant des difficultés de manipulations et un risque très important de perte de matière et d'aggravation des altérations. Les zones les plus fragiles se situent au niveau de la rupture des cervicales à l'origine du détachement complet de la tête ainsi qu'au niveau de l'articulation de la hanche senestre. La peau très sèche et cassante présente un aspect craquelé à l'origine de desquamations ponctuelles. Aucune trace d'infestation active n'a été observée (un traitement par anoxie dynamique à l'azote a été pratiqué en juin 2011 dans les réserves du muséum), toutefois de nombreuses galeries et traces anciennes de trous d'envol sont visibles au niveau du visage, du cou, du thorax, du bras dextre ainsi qu'à l'entrejambe et fragilisent de manière importante les tissus organiques dans ces zones. Des résidus de cadavres d'insectes (Stegobium paniceum) et de larves ont été retrouvés en différents endroits du corps notamment sur le côté dextre de l'abdomen.

L'ensemble du corps est particulièrement empoussiéré et encrassé masquant la lecture des détails de la surface. Cet encrassement constitue par ailleurs un substrat nutritif ayant pu favoriser le développement d'insectes et de micro organismes et participe à l'acidification des textiles déjà oxydés et cassants. Des résidus végétaux vraisemblablement anciens sont observables surtout dans le dos.

Débandelettage mis à part, la momie a subi plusieurs campagnes d'interventions au cours de son histoire matérielle, à la fois dans une tentative de consolidation

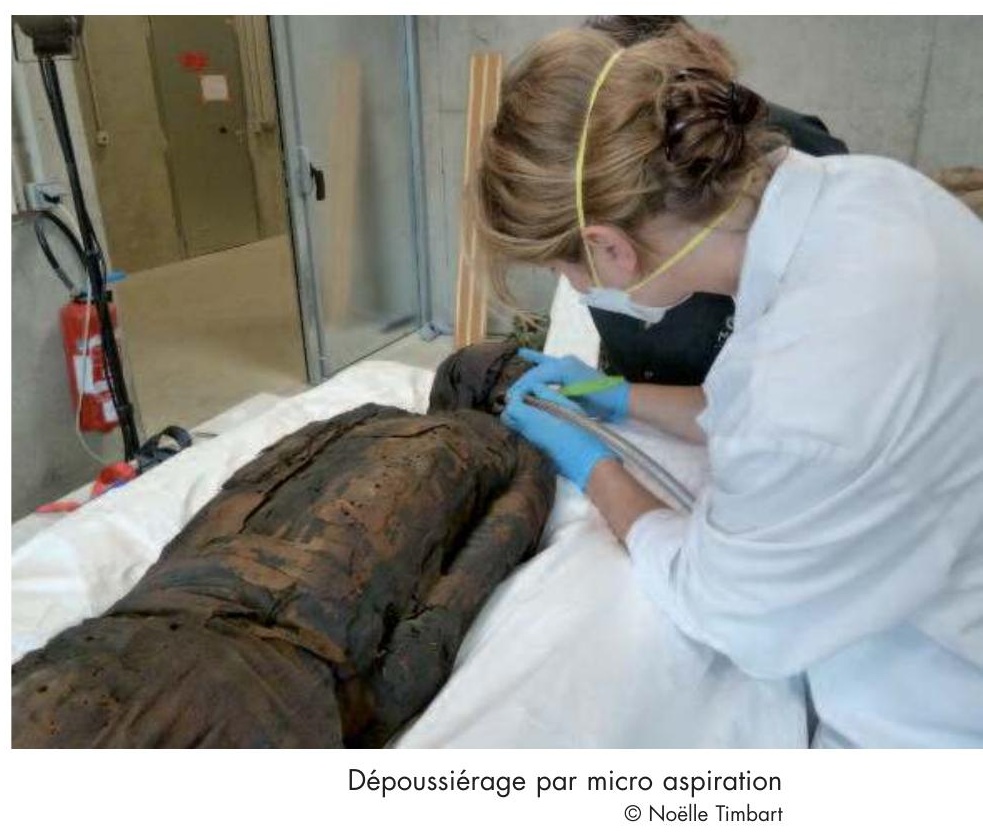


comme l'attestent les différentes traces brillantes d'adhésif au niveau des zones fragilisées et des textiles ainsi que la tige métallique introduite pour solidariser la tête du reste du corps. Des interventions davantage liées à la muséographie et au mode de présentation ont également été pratiquées (percements de fixation au niveau des genoux, bassin, dos, crâne).

\section{Objectifs et déroulement de l'intervention}

L'intervention entreprise sur la momie dans une perspective minimale et non intrusive avait pour objectif d'améliorer son état sanitaire et de procéder à son reconditionnement afin de limiter les manipulations directes au vu de sa fragilité structurelle. En raison du risque trop important de dégradation, il a été proposé de ne pas intervenir sur le montage actuel de la tête qui ne constitue pas à première vue un facteur d'altération sur le court ou le moyen terme.

Avant l'intervention, la momie était disposée dans un cercueil en contre-plaqué peint réalisé dans les années 1970 pour les besoins scénographiques liés à sa présentation. La momie a pu être sortie relativement facilement de son cercueil par l'introduction de sangles sous le plateau disposé au fond de la cuve.

Pour les besoins de l'intervention et de l'étude, il a été procédé au retournement du corps. Pour ce faire, la momie a été manipulée par trois personnes à l'aide de matelas-coques adaptés des modèles médicaux utilisés pour l'immobilisation et le transport des grands blessés. Ceci a permis la réalisation d'un dépoussiérage minutieux de la face et du dos par micro aspiration sans entraîner de tension sur les zones les plus fragiles.

Afin d'assurer le stockage et l'éventuelle présentation au public dans les meilleures conditions possibles, la momie a été placée sur un plateau de présentation et de manipulation en polycarbonate gainé d'une double couche de ouate de polyester et d'une toile de lin naturelle décatie fixée au revers du panneau par des agrafes de tapissier.

La nuque a été calée à l'aide d'un bloc de mousse de polyéthylène (ethafoam ${ }^{\circledR}$ ) découpé en berceau et gainé de toile de lin maintenue par des points de colle acrylique. La momie sur son plateau a été finalement installée dans sa boîte de conditionnement réalisée sur mesure. Celle-ci est constituée d'un fond de caisse avec quatre pans rabattables en polycarbonate renforcés par des cornières en aluminium assemblés par des gonds démontables permettant une parfaite accessibilité à la momie sans avoir besoin de la manipuler directement.

\section{Conclusion}

Entrée en 1819 dans le tout récent musée d'Histoire naturelle de la ville de Nantes, la momie égyptienne a traversé 200 ans d'histoire au cours desquels le regard du public et des professionnels des musées s'est transformé. Entre son retour d'Égypte en tant qu'antiquité et son statut actuel de reste humain, elle porte les traces physiques des traitements qu'elle a subie au cours de différentes époques.

Les compétences complémentaires des différents intervenants ont permis d'enrichir la connaissance du sujet. Les analyses chimiques permettent de confirmer les hypothèses de l'archéologue, l'imagerie médicale renvoie à l'être vivant que fut la momie mais aussi est une aide précieuse avant l'intervention du restaurateur et les décisions du conservateur.

$\mathrm{Si}$ cette momie n'a pas vocation de figurer en exposition permanente au muséum de Nantes en regard du projet scientifique de l'établissement, sa restauration permettra de la présenter lors d'expositions temporaires liées à l'égyptologie. Son conditionnement sur un plateau recouvert de lin facilitera sa manipulation mais aussi permettra une présentation sobre, en position allongée, conformément au respect dû aux restes humains.

Les auteurs tiennent à adresser leurs remerciements au professeur Benoît Dupas chef du service radiologie et imagerie médicale du CHU de Nantes, pour la radiographie et la scanographie de la momie; à Christophe Moreau et son équipe du laboratoire de Mesure du Carbone 14 (LMC14) au CEA de Sacla (C C2RMF, pour les mesures radiocarbone des échantillons; à Gwénaël Lemoine, restauratrice et Jean-Gabriel Aubert, photographe au laboratoire de restauration Arc'antique, pour leurs conseils; à Luc Remy directeur adjoint du muséum de Nantes, pour son aide dans la conduite de cette étude.

\section{Notes}

(1) Mainterot P. Aux origines de l'égyptologie. Voyages et collections de Frédéric Cailliaud (1787-1869). Presses Universitaires de Rennes, 2011.

(2) Archives Cailliaud conservées au muséum d'Histoire naturelle de Nantes, ainsi qu'au musée départemental Dobrée.

(3) Dubuisson F.-R. Muséum d'Histoire naturelle, Journal de Nantes et de la Loire-Inférieure $\mathrm{n}^{\circ} 2347,18$ juin 1819.

(4) Dr Maréschal Rapport sur la momie donnée à la Société académique par M.-F. Cailliaud de Nantes, extrait du Journal de Médecine, Nantes, 1826. (5) Après examen, à l'aide de photographies par infrarouges et ultraviolets, seuls quelques caractères sont identifiables. Il s'agit vraisemblablement 
du chapitre 72 du Livre pour Sortir le Jour, qui se trouve fréquemment inscrit sur les cercueils aux époques tardives.

(6) Flaubert G. Voyage en Bretagne : par les champs et par les grèves. Paris :

éd. Complexe, 1989, p. 89.

(7) Sowada, K., Jacobsen, G.-E., Bertuch, F., Palmer, T. and Jenkinson, A. Who's that lying in my coffin? An imposter exposed by $14 \mathrm{C}$ dating, Radiocarbon, n53, 2011, pp. 221-228.

(8) Hedges R.-E.-M. and Vanklinken G.J. A review of current approaches in the pretreatment of bone for radiocarbon dating by AMS, Radiocarbon, n³4 (3), 1992, pp. 279-291.

(9) Richardin, P., Gandolfo, N., Carminatti, P. and Walter, P.-A New protocol for radiocarbon dating of hair and keratin type samples : Application to an Andean mummy from the National Museum of Natural History in Paris, Archaeological and Anthropological Sciences, n³ (4), 2011, pp. 379-384.

(10) Beavan N., Halcrow S., Mac Fadgen B. et al. Radiocarbon dates from jar and coffin burials of the Cardamon Moutains reveal a unique mortuary ritual in Cambodia's Late to Post-Angkor period (15th-17th centuries AD), Radiocarbon, 54 (1), 2012, pp. 1-22.

(11) Richardin, P., Cuisance, F., Buisson, N., Asensi-Amoros, V. and Lavier, C. AMS radiocarbon dating and scientific examination of high historical value manuscripts: application to two Chinese manuscripts from Dunhuang, Journal of Cultural Heritage, $\mathrm{n}^{\circ} 11,2010$, pp. 398-403.

(12) Richardin, P., Guerra, M.-F. et Salomon, J. LMC14 : le nouveau laboratoire pour la mesure du carbone-14 par spectrométrie de masse par accélérateur, Technè, n ${ }^{\circ}$ 7, 2003, pp. 99-100.

(13) Cottereau, E., Arnold, M., Moreau, C. et al. Artemis, the new 14C AMS at LMC14 in Saclay, France. Radiocarbon, n49 (2), 2007, pp. 291-299.
(14) Mook, W.-G. and Van der Plicht, J. Reporting 14C Activities and Concentrations. Radiocarbon, n41 (3), 1999, pp. 227-239.

(15) Bleton, J., Méjanelle, P., Sansoulet, J. and Tchapla, A. Characterization of neutral sugars and uronic acids after methanolysis and trimethylsilylation for recognition of plant gums. Journal of Chromatography, $\mathrm{n}^{\circ} 720,1996$, pp. $27-49$

(16) Richardin P., Bonnassies-Termes S. and Doré J.-C. Characterization by gas chromatography and multivariable analysis of vegetable gums used as binding media in inks. In MicroAnalysis of Parchments. Glasgow : ed. René Larsen, Archetype Publication Ltd., 2002, pp. 31-44.

(17) Anderson, D.-M.-W. and Karamalla, K.-A. Studies on uronic acid materials. Part XII. The composition of Acacia Gum Exudates. Journal of the Chemical Society, Section C., 8, 762, 1966.

(18) Bleton, J., Méjanelle, P., Sansoulet, J. and Tchapla, A. Characterization of neutral sugars and uronic acids after methanolysis and trimethylsilylation for recognition of plant gums. Journal of Chromatography, n720, 1996, pp. 27-49.

(19) Nussinovitch, A. Chapter 6. Gum Exudates in Water-Based Adhesives, in Nussinovitch, A. Plant Gum Exudates of the World. Sources, Distribution, Properties, and Applications, CRC Press, 2009, pp. 293-310.

(20) Salima Ikram et Aidan Dodson ont recensé différents positionnements pour les époques tardives : il est ainsi fréquent que les deux bras soient ramenés sur le buste, ou bien que le bras gauche soit replié sur la poitrine et le bras droit tendu le long du corps, comme c'est le cas pour le sujet de cette étude, ou encore que les deux mains couvrent les parties génitales. Un exemple de positionnement identique est connu à une époque antérieure sur le cercueil de Tairsekheru (fin XVIIIe-début XIXe, voir Ikram, Dodson, p. 225). 